p53 immunohistochemistry on $39.4 \%$ with a sensitivity of $98.5 \%$ to detect p53abn (99.6\% negative predictive value). Cytologic features including tumor giant cells, smudged chromatin, cherry-red/macronucleoli, and atypical mitoses accurately predicted p53abn. In 7/292, p53abn upgraded ESGO risk groups (2 to intermediate-risk, 5 to high-risk). EEC12/ stage IA patients had an excellent cause-specific 5-year survival of $98.5 \%$.

Conclusions Pathologists can select cases for p53 testing with high sensitivity and low risk of false negativity. Molecular characterization of endometrial carcinomas has great potential to refine ESGO risk classification for a small subset but offers little value for approximately half of endometrial carcinomas, namely, EEC12/stage IA.

\section{EPV104/\#228 EMERGING IMMUNOTHERAPY PARADIGMS IN ADVANCED ENDOMETRIAL CANCER: THE EFFECT OF ONLINE EDUCATION ON CLINICIAN KNOWLEDGE AND CONFIDENCE}

${ }^{1} \mathrm{G}$ Fisher*, ${ }^{1} \mathrm{~A}$ Furedy, ${ }^{1} \mathrm{~J}$ Vandenbroucque, ${ }^{2} \mathrm{~N}$ Colombo. ${ }^{1}$ Medscape, Medical Education, London, UK; ${ }^{2}$ Instituto Europeo di Oncologia, Department of Gynecology, Milan, Italy

\subsection{6/ijgc-2021-IGCS.174}

Objectives This study determined whether online continuing medical education (CME) could improve the knowledge of oncologists (oncs) and obstetricians/gynaecologists (obs/gyns) regarding the rationale and evidence for immunotherapy paradigms in advanced endometrial cancer.

Methods A 30-minute online video lecture was launched for physicians outside the USA August 2020 with data collected to November 2020. Educational effect assessed with repeatedpairs pre-/post-activity- individual participants serving as own control. 3 multiple-choice, knowledge questions and 1 self-efficacy, 5-point Likert scale confidence question were analyzed. Chi-squared test assessed pre- to post-activity change $(5 \%$ significance level, $\mathrm{P}<.05)$. Magnitude of change in total number of correct responses overall, and for each question, determined with Cramer's V $(<.06=$ Modest, $0.06-0.15=$ Noticeable, $0.16-0.26=$ Considerable, $>.26=$ Extensive $)$.

Results $142 \mathrm{obs} / \mathrm{gyns}$ and 60 oncs completed pre- and postactivity questions. Positive educational effect was observed for obs/gyns (noticeable effect, $\mathrm{V}=.092, \mathrm{P}<.01$; average\% of correct responses increasing from 33 to $42 \%$ ) and oncs (noticeable effect, $\mathrm{V}=.150, \mathrm{P}=.0043$; average $\%$ of correct responses increasing from 47 to $62 \%$ ). Increases in correct responses post-activity seen for questions on response to 2 nd line chemotherapy (\% relative improvement, obs/gyn: 23\%, oncs $22 \%$ ), rationale for immunotherapy (obs/gyns: 24\%, oncs: 72\%), data for the dostarlimab GARNET trial (obs/gyns: 36\%, oncs: 21\%). Confidence in knowledge of the evidence for immunotherapy strategies increased post-activity (total average confidence shift: $27 \% \mathrm{obs} / \mathrm{gyns}$ and $40 \%$ oncs). Overall, $22 \%$ of learners' responses were improved and $39 \%$ of learners' responses were reinforced.

Conclusions This online CME activity resulted in a positive educational impact for both clinical specialties. However, education gaps remained evident post-activity.

\section{EPV105/\#237 COMBINATION TARGETED TREATMENT WITH MEK AND PAN-ERBB INHIBITORS ENHANCES ANTITUMOR ACTIVITY IN ERBB AMPLIFIED EX- VIVO SEROUS ENDOMETRIAL CANCER CELLS}

${ }^{1} \mathrm{~A}$ Larish*, ${ }^{2} \mathrm{~W}$-H Lin, ${ }^{3} \mathrm{~J}$ Smadbeck, ${ }^{3} \mathrm{~S}$ Sotiriou, ${ }^{3} \mathrm{~J}$ Cheville, ${ }^{1} \mathrm{~F}$ Harris, ${ }^{1} \mathrm{R}$ Feathers, ${ }^{1} \mathrm{~J}$ Lynch, ${ }^{4} \mathrm{~A}$ Kumar, ${ }^{5} \mathrm{M}$ Block, ${ }^{1} \mathrm{~L}$ Jones, ${ }^{1} \mathrm{G}$ Karagouga, ${ }^{1} \mathrm{~A}$ Mccune, ${ }^{5} \mathrm{~A}$ Mansfield, ${ }^{3} \mathrm{~S}$ Murphy, ${ }^{4} \mathrm{~A}$ Mariani, ${ }^{3} \mathrm{G}$ Vasmatzis, ${ }^{2} \mathrm{P}$ Anastasiadis, ${ }^{5}$ J Weroha. 'Mayo Clinic, Obstetrics and Gynecology, Rochester, USA; ${ }^{2}$ Mayo Clinic, Department of Laboratory Medicine and Pathology, Jacksonville, USA; ${ }^{3}$ Mayo Clinic, Department of Laboratory Medicine and Pathology, Rochester, USA; ${ }^{4}$ Mayo Clinic, Division of Gynaecologic Surgery, Department of Obstetrics and Gynaecology, Rochester, USA; ${ }^{5}$ Mayo Clinic, Medical Oncology, Rochester, USA

\subsection{6/ijgc-2021-IGCS. 175}

Objectives ERBB pathway alterations present therapeutic targets in high grade endometrial cancer (EC), but efficacy can be limited by persistent co-activation of other ERBB binding partners. The efficacy of dual-inhibition MEK+pan-ERBB or $\mathrm{BET}+$ pan-ERBB in an ERBB2/ERBB3 amplified EC was investigated via $3 \mathrm{D}$ microcancer ex-vivo cell assay.

Methods Tumor was prospectively collected from a patient with stage IIIc1 serous EC. Whole exome, mRNA, and MatePair genomic characterization was performed. Tumor cells were grown in $3 \mathrm{D}$ culture and subjected to titrating drug treatments. Cell viability was determined by the CellTiter-Glo Luminescent Assay. Data transformation and dose-response curves were generated using GraphPad PRISM using the variable slope model. CalcuSyn software with the Chou-Talalay method analyzed drug interactions and synergy. Afatinib, binimetanib, and JQ1 were used to inhibit pan-ERBB, MEK1/2, BET, respectively. For translational relevance, inhibitory effect was defined as percent reduction in ATP from baseline at the
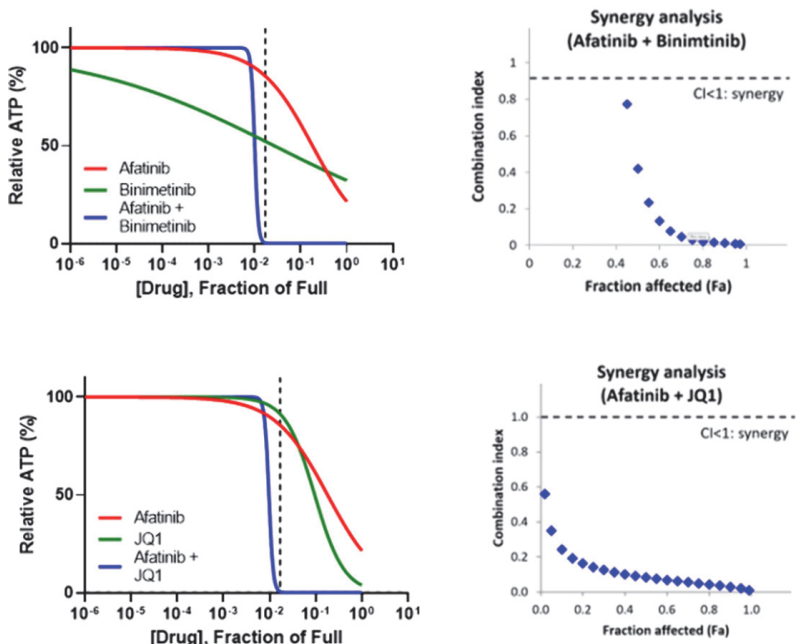

Abstract EPV105/\#237 Figure 1 Microcancer ex vivo exposure to MEK+pan-ERBB inhibitors. Dose response curves of single and combination treatments (left) were 10-fold titrated across 8 log doses for each agent. The highest concentration (i.e. fraction of ful $(\mathrm{FoF})=1$ ) of afatnib, binimetinib and afatinib+JQ1 was $3 \mathrm{uM}, 10 \mathrm{uM}$, and $50 \mathrm{uM}$, respectively. The physiologically achieavable concentration of afatinib is insicated (dotted lines). A comnination index ( $\mathrm{Cl}$, right) was used to assess synergy with afatinib+binimtinib and afatinib+JQ1 as shown by $\mathrm{Fa}-\mathrm{Cl}$ plots. 
physiologically achievable concentration (maximum plasma concentration (Cmax) value).

Results Sequencing revealed amplifications of ERBB2 (17q12), RAF1, c-myc, and ERBB3 (12q13.2) low-level gain. Inhibition of viability was moderate by single agents: Afatinib, binimetanib, JQ1, as shown by inhibitory effect values of $14.4 \%, 47.8 \%, 8.8 \%$, respectively at physiologically achievable concentrations (Cmax) of afatinib. Combinations demonstrated increasing inhibitory effect values: $99.7 \%$ for Afatinib + binimetanib, and 99.5\% for Afatinib+JQ1. Synergy was evidenced for both combinations by a combination index $<1$ (figure 1). Conclusions Combined inhibition of pan-ERBB with inhibition of MEK or BET proteins synergistically suppress viability in patient-derived serous EC harboring ERBB amplifications.

\section{EPV106/\#249 ENDOMETRIAL CANCER IMMUNOHISTOCHEMICAL RISK STRATIFICATION IN A LARGE UTERINE- CONFINED CANCER SERIES}

${ }^{1}$ E Perrone*, ${ }^{2} \mathrm{~F}$ De Felice, ${ }^{3}$ I Capasso, ${ }^{4} \mathrm{D}$ Arciuolo, ${ }^{1} \mathrm{E}$ Distefano, ${ }^{1} \mathrm{D}$ Lorusso, ${ }^{4} \mathrm{GF}$ Zannoni, ${ }^{1} \mathrm{G}$ Scambia, ${ }^{1} \mathrm{~F}$ Fanfani. ${ }^{1}$ Fondazione Policlinico Universitario A. Gemelli, IRCCS, Uoc Ginecologia Oncologica, Dipartimento Per La Salute Della Donna E Del Bambino E Della Salute Pubblica, Rome, Italy; 'Policlinico Umberto I, 'Sapienza' University of Rome, Department of Radiotherapy, Rome, Italy; ${ }^{3}$ Policlinico universitario Agostino Gemelli, Uoc Ginecologia Oncologica, Roma, Italy; ${ }^{4}$ Fondazione Policlinico Universitario A. Gemelli, IRCCS, Gyneco-pathology and Breast Pathology Unit, Dipartimento Per La Salute Della Donna E Del Bambino E Della Salute Pubblica, Rome, Italy

\subsection{6/ijgc-2021-IGCS.176}

Objectives The aim of this study is to assess the clinical reproducibility and the oncological validity of the Endometrial cancer (EC) risk stratification based on the molecular information given by the immunohistochemistry (IHC).

Methods Retrospective IHC analyses were conducted in a large series of 778 pre-operative uterine-confined ECs, studying the presence/absence of MLH1, MSH2, MSH6, to define the mismatch repair (MMR) stable or instable phenotype; the presence of p53 mutations and other molecular features. The molecular profile was correlated with histological, clinical and prognostic EC patients' data.

Results Based on the IHC, we defined 3 EC populations: MMR stable (MMRs), instable (MMRi) and p53 mutated $(\mathrm{p} 53+)$ patients. Our result demonstrated that the IHC stratification statistically correlated with the most relevant anatomoclinical features: FIGO stage $(p<0.001)$, grading $(12,5 \%$ G3 in MMRs vs $22.9 \%$ in MMRi vs $95.3 \%$ in $\mathrm{p} 53+, \mathrm{p}<0.001)$, histotype (Type II 6.2\% in MMRs vs $5.3 \%$ in MMRi vs $87.5 \%$ in $\mathrm{p} 53+, \mathrm{p}<0.001$ ), presence of LVSI (positive in $16.3 \%$ in MMRs vs $23.8 \%$ in MMRi vs $38.7 \%$ in $\mathrm{p} 53+$, $\mathrm{p}<0.001$ ), myometrial invasion and tumor dimension $(\mathrm{p}=0.003$ and $\mathrm{p}<0.001$ respectively). Again, the 3 IHC populations statistically reflected the EC risk class ESGO-ESMOESP classification $2020 \quad(\mathrm{p}<0.001)$. These results were confirmed also in Kaplan-Meier curves in terms of over-all survival $(\mathrm{OS})$ and disease-free survival (DFS) $(\mathrm{p}<0.001)$ (figure 1).

Conclusions In this large series, we demonstrated that the pragmatic and systematic use of IHC may have an important role to properly stratify, in terms of histological features and clinical outcome, the uterine-confined EC patients.

\section{EPV107/\#258 ENGOT-EN11/GOG-3053/KEYNOTE-B21: PHASE 3 STUDY OF PEMBROLIZUMAB OR PLACEBO IN COMBINATION WITH ADJUVANT CHEMOTHERAPY WITH/WITHOUT RADIOTHERAPY IN PATIENTS WITH NEWLY DIAGNOSED HIGH-RISK ENDOMETRIAL CANCER}

${ }^{1} \mathrm{~T}$ Van Gorp ${ }^{*},{ }^{2} \mathrm{M}$ Mirza, ${ }^{3} \mathrm{~A}$ Lortholary, ${ }^{4}$ I Vergote, ${ }^{5} \mathrm{D}$ Cibula, ${ }^{6} \mathrm{~A}$ Walther, ${ }^{7} \mathrm{~A}$ Savarese, ${ }^{8} \mathrm{M}$-P Barretina-Ginesta, ${ }^{9} \mathrm{~F}$ Ortaç, ${ }^{10} \mathrm{C}$ Papadimitriou, ${ }^{11} \mathrm{~L}$ Bodnar, ${ }^{12} \mathrm{C}-\mathrm{H}$ Lai, ${ }^{13} \mathrm{~K}$ Hasegawa, ${ }^{14} \mathrm{X}$ Xie, ${ }^{15} \mathrm{EL}$ Barber, ${ }^{16} \mathrm{RL}$ Coleman, ${ }^{17} \mathrm{~S}$ Keefe, ${ }^{17} \mathrm{R}$ Orlowski, ${ }^{18} \mathrm{~B}$ Slomovitz. ${ }^{1}$ UZ Leuven, Gynaecological Oncology, Leuven, Belgium; ${ }^{2}$ NSGO-CTU and Rigshospitalet, Copenhagen University Hospital, Copenhagen, Denmark; ${ }^{3}$ Centre Catherine de Sienne, Hôpital Privé Du Confluent, Nantes, France; ${ }^{4} B G O G$ and University Hospitals Leuven, Leuven Cancer Institute, Leuven, Belgium; ${ }^{5}$ Department of Obstetrics and Gynecology, General Faculty Hospital In Prague, First Faculty of Medicine, Charles University, Prague, Czech Republic; ${ }^{6}$ Bristol Cancer Institute, University Hospitals Bristol, Bristol, UK; ${ }^{7}$ Department of Medical Oncology, Istituto Nazionale Tumori Regina Elena, Rome, Italy; ${ }^{8}$ Catalan Institute of Oncology and Girona Biomedical Research Institute, Medical School University of Girona, Girona, Spain; ${ }^{9}$ Department of Obstetrics and Gynecology, Ankara University School of Medicine, Ankara, Turkey; ${ }^{10}$ Aretaieio University Hospital, National and Kapodistrian University of Athens, Athens, Greece; ${ }^{11}$ Department of Oncology and Immunooncology, Warmian-masurian Cancer Center of The Ministry of The Interior and Administration's Hospital, Olsztyn, Poland; ${ }^{12}$ TGOG and Department of Gynecology and Obstetrics, Chang Gung Memorial Hospital, Linkou Branch, Taoyuan, Taiwan; ${ }^{13}$ Department of Gynecologic Oncology, Saitama Medical University, Hidaka, Saitama Prefecture, Japan; ${ }^{14}$ Women's Hospital, School of Medicine, Zhejiang University, Hangzhou, China; ${ }^{15}$ Department of Gynecologic Oncology, Northwestern University Feinberg School of Medicine, Chicago, USA; ${ }^{16}$ Department of Gynecologic Oncology, Us Oncology Research, The Woodlands, USA; ${ }^{17}$ Oncology, Merck and Co., Inc., Kenilworth, USA; ${ }^{18}$ Department of Gynecologic Oncology, Broward Health, Fort Lauderdale, USA

\subsection{6/ijgc-2021-IGCS.177}

Objectives Pembrolizumab, an anti-PD-1 antibody, has demonstrated activity as monotherapy and in combination with lenvatinib in patients with previously treated mismatch repair (MMR) deficient and MMR proficient endometrial cancer (EC). ENGOT-en11/GOG-3053/KEYNOTE-B21 (NCT04634877) is a phase 3, randomized, double-blind study of pembrolizumab or

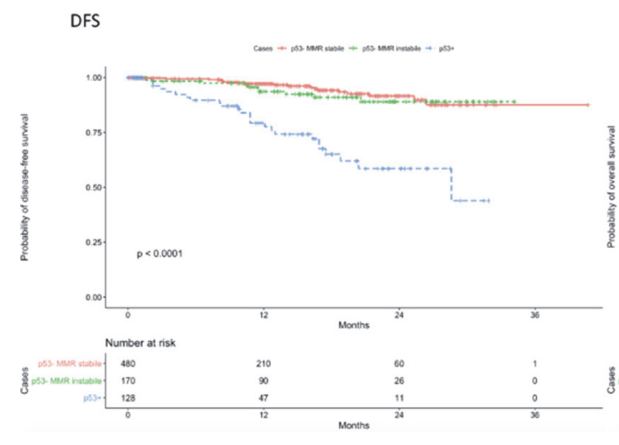

os

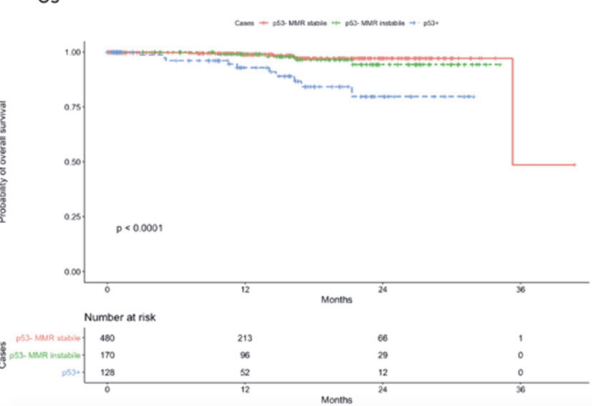

